\title{
Neurofibroma of Spindle Cell Origin, a Diagnostic Dilemma to General Dentist.
}

\author{
Dr. Swapnil Moghe ${ }^{1}$,Dr. M.K. Gupta ${ }^{2}$,Dr. Mrinal Sathpathy ${ }^{3}$ Dr. Ajay Pillai ${ }^{4}$, \\ Dr. Saksham Nahar ${ }^{5}$, \\ ${ }^{I}$ (Reader, Department Of Oral \& Maxillofacial Surgery, Peoples Dental Academy, Peoples University, Bhopal, \\ Madhya Pradesh, India.) \\ ${ }^{2}$ (Professor, H.O.D. \& Dean, Department Of Oral \& Maxillofacial Surgery, Peoples Dental Academy, Peoples \\ University, Bhopal, Madhya Pradesh, India.) \\ 3 ( Sr. Lecturer, Department Of Oral \& Maxillofacial Surgery, Peoples Dental Academy, Peoples University, \\ Bhopal, Madhya Pradesh, India.) \\ ${ }^{4}$ (Reader, Department Of Oral \& Maxillofacial Surgery, Peoples Dental Academy, Bhopal, India.) \\ ${ }^{5}$ (Post graduate Student, Department Of Oral \& Maxillofacial Surgery, Peoples Dental Academy, Bhopal, \\ India.)
}

\begin{abstract}
Neurofibromas arise from a mixture of cell types including Schwann cells and perineural fibroblasts. They may occur as solitary lesions or in association with neurofibromatosis. Although most commonly reported in soft tissues, neurofibromas do occur in bone. And very few cases have been reported in association with the inferior alveolar nerve. We report a case of neuro-fibroma of spindle cell origin associated with the inferior alveolar nerve in a 22 year old man. Pain or paresthesia may result from lesions of the inferior alveolar nerve. Patients presents with cortical expansion. Intra-osseous lesions may produce a well demarcated or poorly defined unilocular or multilocular radiolucency. Adjacent soft tissue neurofibromas may produce cortical erosion. Solitary neurofibromas and those found in association with neurofibromatosis share the same microscopic features ${ }^{l}$. The tumor is composed of spindle-shaped cells with fusiform or wavy nuclei in a delicate connective tissue matrix. It is not encapsulated and may blend with the adjacent connective tissues. The normally recommended treatment of solitary lesions following biopsy is localized excision.
\end{abstract}

Key Words: Neurofibroma, Spindle cell, Tumor

Case presentation:

\section{Introduction}

The patient reported a slow growing lesion in lower left mandibular region extending from canine to $2^{\text {nd }}$ molar region. A 22-year-old man presented to the department of the Oral and Maxillofacial Surgery with a 1 month history symptom of paraesthesia of lower left side of mandible extending from corner of mouth to angle of mandible. His medical history was unremarkable and there was no history of gum-related disease or trauma to the maxillofacial complex. Mobility of teeth was absent, also, a history of numbness of the lower lip since 2 months with no history of extraoral swelling was significant. An intraoral examination revealed a obliteration of muco-buccal fold of about $3 \times 1 \mathrm{~cm}$ in the left lower canine- molar region with no signs of ulceration (Figure 1). On palpation, the swelling was firm in consistency with underlying bone from left canine to molar region. No neck nodes were palpable and the cranial examination was normal. Funelling \& widening of inferior alveolar canal was evident on OPG. (Figure 2) 


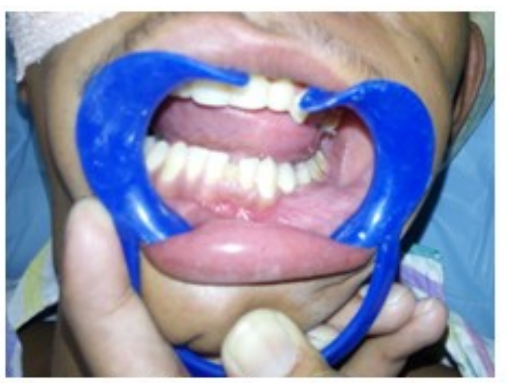

Figure 1: Obliteration of muco-buccal fold With 33-36 region.

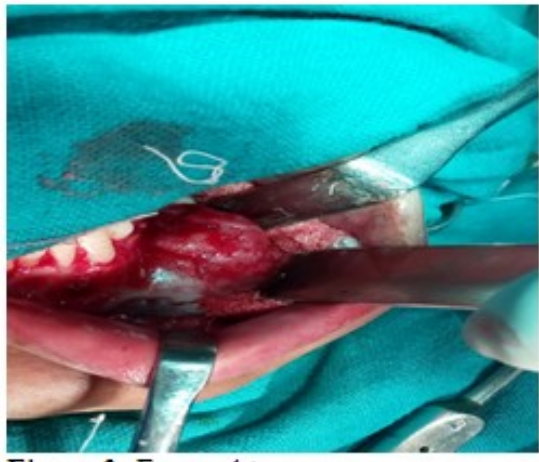

Figure 3: Exposed tumor mass

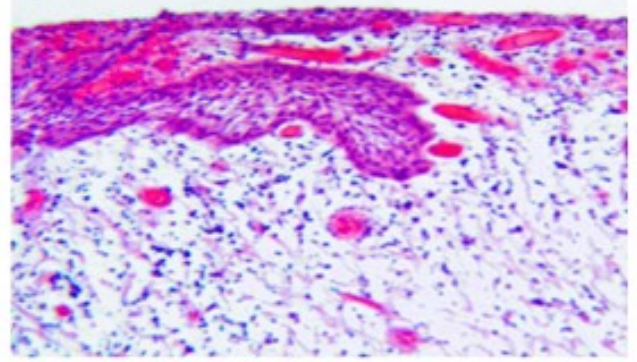

Figure 5: Histopathology shows a tumor of Proliferative spindle cells with a stroma of Irregular collagen fibers $(\mathrm{HE}, \times 100)$.

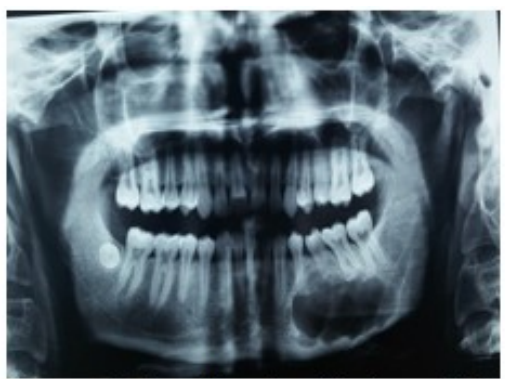

Figure 2: Funelling \& widening of inferior alveolar canal

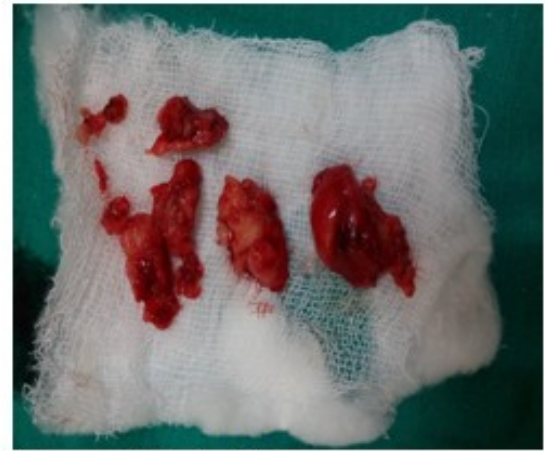

Figure 4: Excised Tumor mass.

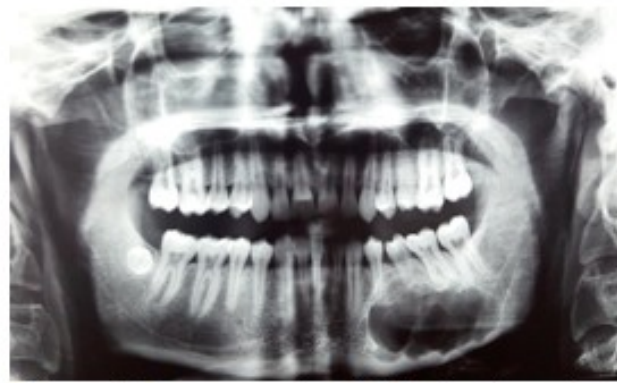

Figure 6: Post -operative OPG after 6 months.

Not many cases have been reported in the literature for the same. Under all aseptic conditions, the patient was intubated under G.A. \& local anesthetic was infiltrated around lower anterior \& posterior mandibular region on the left side. A crevicular incision was placed from the lower left side central incisor till second molar with bilateral releasing incisions. A full thickness muco-periosteal flap was raised, the tumor mass was exposed (Figure 3) \& through the opened window, the tumor mass was removed (Figure 4). Nerve avulsion was done to remove the remnants of IAN. The surgical site was irrigated with betadine \& saline. Hemostasis was achieved \& closure was done with 3-0.

\section{Investigations}

Orthopantamogram revealed an osteolytic scalloped lesion extending from the lower left canine region to the second molar. The lesion also shows erosion of buccal cortical plate.

Microscopically the tumor is composed of an irregular pattern of proliferative spindle cells (Figure 5). The stroma is composed of collagen fibers and mucoid masses. Small axons all over the tumoral tissue are demonstrated with silver staining.

\section{Treatment \& follow up:}

The patient was followed up for 6 months (Figure 6). The postoperative OPG showed good healing with no signs of recurrence at the surgical site. 


\section{Discussion:}

Neurofibroma (NF) is a benign tumor of neural origin derived from the peripheral nerve sheath that may have variable histology. Nerve sheath tumors located in the jaw are extremely rare, having published only a few cases of central neurofibroma of the mandible. There is no sex predilection and average age of occurrence is 28 years. In our case it was 22 years old man.

Ninety percent of the neurofibromas are associated with neurofibromatosis ${ }^{2}$ type 1 , so the physical examination and family history should be elicited to exclude the disease. In this case, there were no clinical signs or family history suggestive of neurofibromatosis. The lesion was a solitary one.

Hubner and Lewis ${ }^{3}$ developed an animal model to investigate the causative factors in the development of the lesion. They reported that the peripheral nerve section resulted in the formation of an expanded connective tissue cap at the end of the proximal segment. Nerve fibers attempting to re-establish continuity with the distal segment penetrated into and beyond the cap, becoming tangled and entrapped in the soft tissue. But in our case, there was no history of trauma.

In the mandible, the lesions most commonly arise from the mandibular nerve with accompanying pain and par aesthesia. In such cases the radiograph shows flaring of mandibular foramen, the so called “blunderbuss' foramen or fusiform enlargement of the mandibular canal, as was seen in our case ${ }^{4}$.

Histologically the tumor is composed of spindle cells arranged in bundles within collagen and mucopolysaccharides matrix that makes the tumor soft \& even gelatinous. The nerve fibres are within the lesion. The tri-chrome stains like Mallory's or Masson's may be useful in identifying collagen. Alcian blue stain is helpful in staining perineural mucin which is not present in scar tissue.

The lesion should be differentiated with schwanomma (Antoni A and Antoni B areas) and perineuroma (pattern similar to onion bulbs), as proposed by $\mathrm{Ide}^{5}$.The lesion should also be differentiated from other fibroossoeous lesions like Juvenile ossifying fibroma,Peripheral giant cell granuloma, as reported by Moghe $\mathrm{S}$ etal $^{6,7}$.These lesions do present with diversifying features.

The solitary intraosseous neurofibroma may be the first manifestation of neurofibromatosis. It is important to put patient on regular follow-up \& correlating clinically \& radiographically, since recurrence and malignant changes have been reported ${ }^{8}$.

\section{References}

[1]. Zachariades N, Mezitis M, Vairaktaris E, Triantafyllou D, Skoura- Kafoussia C, Konsolaki-Agouridaki E, Hadjiolou E, Papavassiliou D: Benign neurogenic tumors of the oral cavity. Int J Oral Maxillofac Surg 1987, 16:70-76.

[2]. Sharma P, Narwal A, Rana AS, Kumar S. Intraosseous neurofibroma of maxilla in a child. J Indian Soc Pedod Prev Dent. 2009; 27: $62-4$.

[3]. HUB E. Amputation neuromas: Their development and prevention. Archives of Surgery 1920; 1(1):85.

[4]. Rajendran R, Sivapada Sundaram B. Benign and malignant tumors of the oral cavity. Shafer, Hine, Lavy, editors Shafer's Text book of Oral Pathology India: Elsevier2009:120-7.

[5]. Ide F, Shimoyama T, Horie N, Kusama K. Comparative ultrastructural and immunohistochemical study of perineurioma and neurofibroma of the oral mucosa. Oral Oncol. 2004; 40: 948-53.

[6]. Moghe S etal, Juvenile psammomatoid Ossifying Fibroma, IOSR Journal of Dental and Medical Sciences (IOSR-JDMS), Volume 13, Issue 6 Ver. V (Jun. 2014), PP 43-45.

[7]. Moghe S etal, Peripheral Giant Cell Granuloma: A Case Report and Review of Literature, People's Journal of Scientific Research ,Vol. 6(2), July 2013.

[8]. Mori H, Kakuta S, Yamaguchi A, Nagumo M. Solitary intraosseous neurofibroma of the maxilla: report of a case. J Oral Maxillofac Surg. 1993; 51:688-90. 